\title{
Release of phospholipase $\mathrm{C} \zeta$ and $\left[\mathrm{Ca}^{2+}\right]_{i}$ oscillation-inducing activity during mammalian fertilization
}

\author{
Sook-Young Yoon and Rafael A Fissore \\ Department of Veterinary and Animal Sciences, University of Massachusetts, Amherst, Massachusetts 01003, USA \\ Correspondence should be addressed to R A Fissore at Department of Veterinary and Animal Sciences, University of Massachusetts, \\ 411 Paige Laboratories, 161 Holdsworth Way, Amherst, Massachusetts 01003, USA; Email: rfissore@vasci.umass.edu
}

\begin{abstract}
During fertilization of mammalian eggs a factor from the sperm, the sperm factor (SF), is released into the ooplasm and induces persistent $\left[\mathrm{Ca}^{2+}\right]_{i}$ oscillations that are required for egg activation and embryo development. A sperm-specific phospholipase C (PLC), PLC $\zeta$, is thought to be the SF. Here, we investigated whether the SF activity and PLC $\zeta$ are simultaneously and completely released into the ooplasm soon after sperm entry. To accomplish this, we enucleated sperm heads within 90 min of intracytoplasmic sperm injection (ICSI) and monitored the persistence of the $\left[\mathrm{Ca}^{2+}\right]_{i}$ oscillations in eggs in which the sperm had been withdrawn. We also stained the enucleated

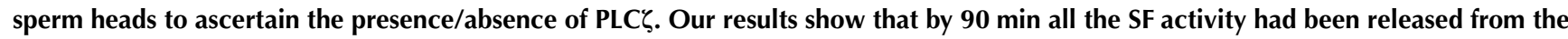
sperm, as fertilized enucleated eggs oscillated as fertilized controls, even in cases in which oscillations were prolonged by arresting eggs at metaphase. In addition, we found that the released SF activity became associated with the pronucleus (PN), as induction of PN envelope breakdown evoked comparable $\left[\mathrm{Ca}^{2+}\right]_{i}$ responses in enucleated and non-manipulated zygotes. Lastly, we found that PLC $\zeta$ localized to the equatorial area of bull sperm and to the post-acrosomal region of mouse sperm and that by 90 min after ICSI all the

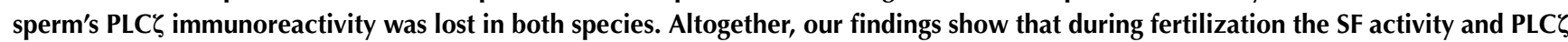
immunoreactivity are simultaneously released from the sperm, suggesting that PLC $\zeta$ may be the only $\left[\mathrm{Ca}^{2+}\right]_{i}$ oscillation-inducing factor of mammalian sperm.
\end{abstract}

Reproduction (2007) 134 695-704

\section{Introduction}

Mammalian eggs are ovulated arrested at the metaphase stage of the second meiotic division (MII). Upon fertilization, the sperm triggers repetitive and persistent changes in the intracellular concentrations of free calcium $\left(\left[\mathrm{Ca}^{2+}\right]_{\mathrm{i}}\right)$, also referred to as $\left[\mathrm{Ca}^{2+}\right]_{\mathrm{i}}$ oscillations, that are required for egg activation (Jones et al. 1995, Schultz \& Kopf 1995, Day et al. 2000). Egg activation entails the progressive initiation of several events, such as cortical granule exocytosis, resumption of meiosis and exit from MII arrest, extrusion of the second polar body and pronucleus (PN) formation (Schultz \& Kopf 1995). The orderly completion of these events ensures the initiation of normal embryo development (Ducibella et al. 2002).

$\left[\mathrm{Ca}^{2+}\right]_{i}$ oscillations are thought to unfold following the release into the ooplasm of a sperm-specific component, the sperm factor (SF), immediately after fusion of the gamete's membranes (Swann 1990, 1996, Stricker 1999). Initial evidence for this hypothesis stemmed from demonstrations that direct injection of sperm into the ooplasm, which circumvented sperm-egg interactions, resulted in normal embryo development and $\left[\mathrm{Ca}^{2+}\right]_{i}$ oscillations (Palermo et al. 1992, Nakano et al. 1997, Sato et al. 1999). Parallel and subsequent studies making use of sperm extracts from several species also found that injection of these extracts caused $\left[\mathrm{Ca}^{2+}\right]_{i}$ oscillations in oocytes of widely diverse species (Swann 1990, Stricker 1997, Wu et al. 1997, Kyozuka et al. 1998, Dong et al. 2000, Tang et al. 2000, Yamamoto et al. 2001), and even in somatic cells (Berrie et al. 1996). Studies to unveil the ontogeny of this activity demonstrated that appearance of SF activity, i.e. the ability to initiate $\left[\mathrm{Ca}^{2+}\right]_{i}$ Oscillations, was not seen until late stages of spermiogenesis (Parrington et al. 2000, Yazawa et al. 2000). Moreover, biochemical studies conducted to ascertain the distribution of SF in the sperm suggested that it localized to the sperm perinuclear theca (PT; Kimura et al. 1998, Perry et al. 1999, 2000), which is a matrix-like multiprotein complex material surrounding the sperm nuclear membrane, and that reportedly represents the first sperm domain to mix with the ooplasm during fertilization (Sutovsky et al. 2003). Nonetheless, the precise distribution of SF in mammalian sperm and its molecular composition remain to be fully elucidated. 
Progress toward identification of the molecular nature of the mammalian SF has been facilitated by evidence showing that sperm-induced $\left[\mathrm{Ca}^{2+}\right]_{i}$ oscillations are underpinned by activation of the phosphoinositide (PI) pathway. A major signaling product of this pathway, inositol 1,4,5-trisphosphate $\left(\mathrm{IP}_{3}\right)$, results from the hydrolysis of phosphatidylinositol 4,5-bisphosphate $\left(\mathrm{PIP}_{2}\right)$ by phospholipase(s) $\mathrm{C}(\mathrm{PLC}) . \mathrm{IP}_{3}$ binds and gates the $\mathrm{IP}_{3}$ receptors $\left(\mathrm{IP}_{3} \mathrm{Rs}\right)$ on the endoplasmic reticulum, the intracellular $\mathrm{Ca}^{2+}$-store, thereby stimulating $\mathrm{Ca}^{2+}$ release. Evidence that the PI pathway is active in mammalian fertilization emanates from studies showing that sperm or SF-induced $\left[\mathrm{Ca}^{2+}\right]_{i}$ oscillations and egg activation are abolished by functional blocking $\mathrm{IP}_{3} \mathrm{R}-1$ antibodies (Miyazaki et al. 1992, Xu et al. 1994), or by treatment with the PLC inhibitor U73122 (Dupont et al. 1996, Jones et al. 1998a, Lee \& Shen 1998, Wu et al. 2001). While a priori one of several PLC isoforms (Rhee \& Bae 1997) present in sperm could be the active PLC, and therefore the SF, the discovery of a sperm-specific PLC, PLC (Saunders et al. 2002), points to it as the putative SF (for review, see Kurokawa et al. 2004, Swann et al. 2004, 2006). Accordingly, injection of mouse PLC $\zeta$ cRNA into mouse eggs induced fertilization-like $\left[\mathrm{Ca}^{2+}\right]_{i}$ oscillations and promoted embryo development to the blastocyst stage (Saunders et al. 2002, Kouchi et al. 2004). In keeping with this finding, sperm extracts depleted of PLC $\zeta$ lacked the ability to initiate $\left[\mathrm{Ca}^{2+}\right]_{i}$ oscillations (Saunders etal. 2002), and reducing the sperm's PLC $\zeta$ content by a transgenic RNA interference (RNAi) approach perturbed fertilizationassociated $\left[\mathrm{Ca}^{2+}\right]_{i}$ oscillations and reduced litter size (Knott et al. 2005). Importantly, expression of PLC $\zeta$ cRNAs

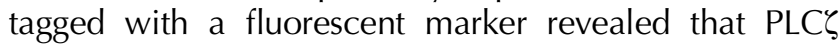
accumulates in the PN (Larman et al. 2004, Yoda et al. 2004, Sone et al. 2005), which is consistent with the finding that, during interphase, the SF activity in fertilized mouse zygotes associates exclusively with either PN (Kono et al. 1996, Knott et al. 2003). Collectively, the data suggest that PLC $\zeta$ is the SF that underlies $\left[\mathrm{Ca}^{2+}\right]_{i}$ oscillations in the mouse and in mammals. Nevertheless, whether PLC $\zeta$ represents the only $\left[\mathrm{Ca}^{2+}\right]_{i}$ oscillation-inducing factor and whether its release renders sperm incompetent to induce $\left[\mathrm{Ca}^{2+}\right]_{i}$ oscillations and egg activation remains to be demonstrated.

Our previous studies showed that within 15 to $60 \mathrm{~min}$ of fertilization, a significant portion of the sperm's $\left[\mathrm{Ca}^{2+}\right]_{i}$ oscillatory activity became dissociated from the sperm and, seemingly, by $120 \mathrm{~min}$ the sperm lost all its ability to induce oscillations (Knott et al. 2003). In this study, we expand on those findings by investigating whether the released SF supports long-term $\left[\mathrm{Ca}^{2+}\right]_{i}$ oscillations equivalent to those seen in fertilization. We also examined whether the released SF achieves association with the PN. Finally, we determined the localization of PLC $\zeta$ in mouse and bull sperm, and investigated whether its release from the equatorial region of the sperm head coincides with loss of the sperm's ability to induce $\left[\mathrm{Ca}^{2+}\right]_{i}$ oscillations.

\section{Results \\ Complete release of $S F$ from the sperm by $90 \mathrm{~min}$ post sperm entry}

In a previous study, we have shown that the fertilizationassociated $\left[\mathrm{Ca}^{2+}\right]_{i}$ oscillatory activity of mouse and bull sperm was mostly released into the ooplasm within 60-120 min post-ICSI or post-IVF, as re-injection of the same sperm into a new egg was unable to initiate oscillations (Knott et al. 2003). However, a question left unanswered was whether the oscillations initiated prior to enucleation were able to persist as long as of those in zygotes in which the sperm was left undisturbed, which would confirm the notion that all the SF activity is released within the first $2 \mathrm{~h}$ of sperm entry. To answer this question, ICSI, sperm enucleation and $\left[\mathrm{Ca}^{2+}\right]_{i}$ monitoring were performed in the time line shown in Fig. 1. To ensure that manipulation of the zygotes was not affecting $\left[\mathrm{Ca}^{2+}\right]_{i}$ responses, comparable amounts of ooplasm were removed from control zygotes. As shown in Fig. $1 \mathrm{~A}$ and $\mathrm{B}$, the $\left[\mathrm{Ca}^{2+}\right]_{i}$ oscillations initiated by ICSI were undisturbed by removal of a sperm-like portion of ooplasm (Fig. 1B), although all these examined zygotes showed the expected decline in frequency as interphase neared (Kono et al. 1996, Day et al. 2000, Larman et al. 2004, Lee et al. 2006). Importantly, removal of the sperm head $90 \mathrm{~min}$ post-ICSI did not affect the pattern or persistence of $\left[\mathrm{Ca}^{2+}\right]_{i}$ oscillations, as nearly all enucleated zygotes (43/50; $87.2 \% \pm 3.2$ (S.E.M)) oscillated for a few hours until oscillations began tailing off as progression into interphase occurred (Fig. 1C, $P>0.05)$. While these results are suggestive of significant release of the SF activity into the ooplasm, they do not necessarily answer whether all the sperm's $\left[\mathrm{Ca}^{2+}\right]_{\mathrm{i}}$ inducing activity is released during this period. This is so because it is well known that if arrested into a metaphase-like stage, fertilized eggs can continue to oscillate for nearly $20 \mathrm{~h}$ (Day et al. 2000), whereas under natural conditions, the oscillations cease by the approximate time of $\mathrm{PN}$ formation (Jones et al. 1995). Hence, to extend these findings, we performed that same experimental design but in the presence of $100 \mathrm{ng} / \mathrm{ml}$ colcemid, which is known to prevent MII exit (Jones et al. 1995, Moses \& Kline 1995, Gordo et al. 2002). Under these conditions, oscillations in enucleated eggs persisted in excess of $8 \mathrm{~h}$ (Fig. 2B and E), which was comparable to the duration of oscillations in control zygotes (Fig. 2A, C and D). Altogether, the data show that within 90 min following fertilization, the totality of the SF activity becomes uncoupled from the sperm to seek its substrate. 

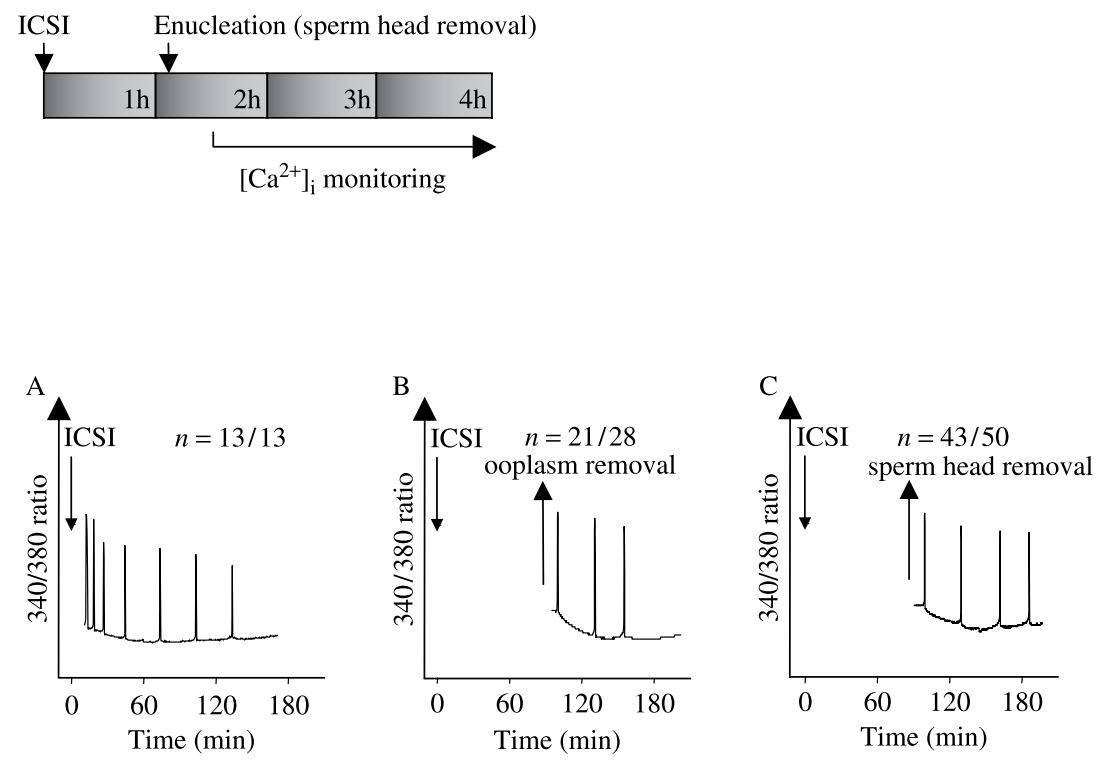

Figure 1 Sperm enucleation after ICSI does not impact the initiation or persistence of $\left[\mathrm{Ca}^{2+}\right]_{i}$ oscillations prior to $\mathrm{PN}$ formation. $\left[\mathrm{Ca}^{2+}\right]_{\mathrm{i}}$ profiles of eggs that underwent control ICSI (A), or from eggs in which ooplasm (B) or the sperm head (C) was removed. Enucleation here and throughout the manuscript was performed at $90 \mathrm{~min}$ postICSI. Following ICSI, eggs were cultured in KSOM for $60 \mathrm{~min}$ prior to enucleation. After enucleation, $\left[\mathrm{Ca}^{2+}\right]_{i}$ monitoring was performed for $\sim 180 \mathrm{~min}$. The scheme representing the time line for the each of the procedures performed to complete the experiment is shown above the $\left[\mathrm{Ca}^{2+}\right]_{i}$ panels. Vertical arrows denote time of ICSI or sperm enucleation.

\section{Released SF associates with the PN during interphase in enucleated zygotes}

In mouse zygotes, $\left[\mathrm{Ca}^{2+}\right]_{i}$ oscillations are entrained with the cell cycle, becoming undetectable by the time zygotes proceed into interphase and form PNs (Ogonuki et al. 2001, Larman et al. 2004, Lee et al. 2006). At the conclusion of this stage, a rise in $\left[\mathrm{Ca}^{2+}\right]_{i}$ seems to accompany the PN envelope breakdown (PNBD), an event that portents the impeding first mitosis (Jones et al. 1995, Day et al. 2000, Gordo et al. 2002, FitzHarris et al. 2003). While this PNBD-associated $\left[\mathrm{Ca}^{2+}\right]_{\mathrm{i}}$ rise is not observed in all fertilized zygotes (Day et al. 2000, Gordo et al. 2002) and its functional significance remains unknown (FitzHarris et al. 2003), it is only detectable in fertilized zygotes, which is consistent with the evidence that after fertilization, the SF activity associates almost exclusively with the PNs (Marangos et al. 2003). Hence, the PNBD-associated $\left[\mathrm{Ca}^{2+}\right]_{i}$ rise could be used as an indicator of the cellular distribution of the SF. Hence, we wished to ascertain whether

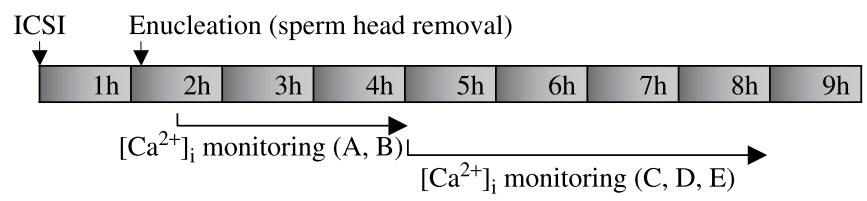

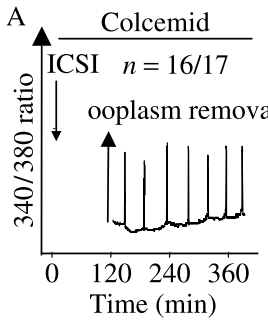
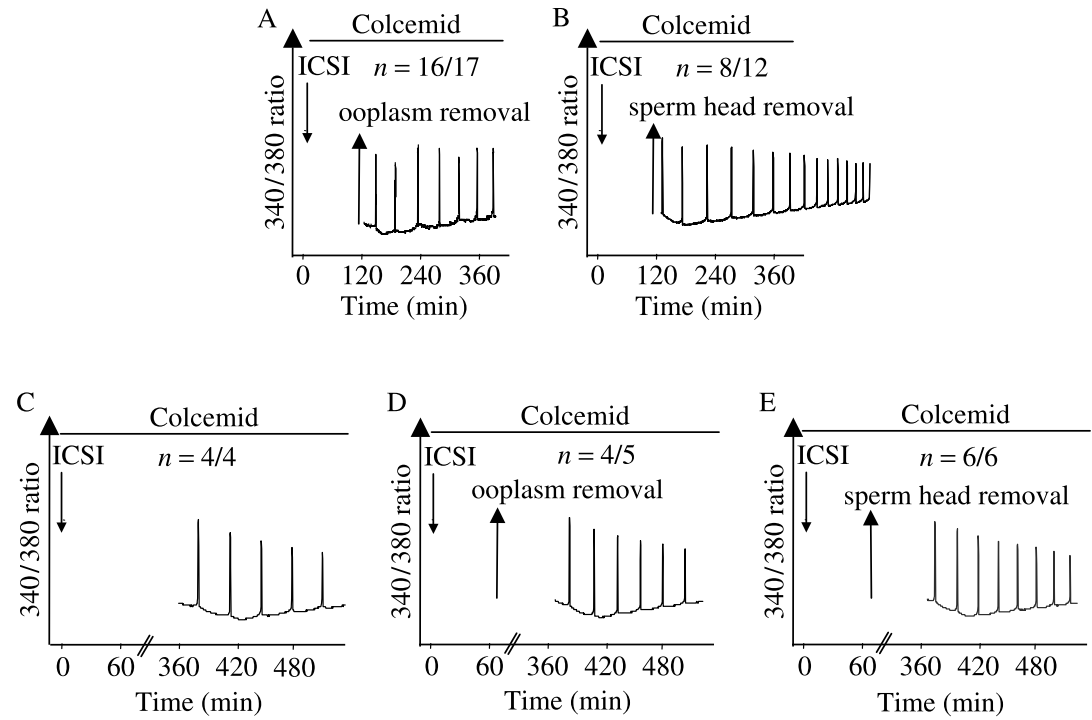

Figure 2 Sperm enucleation does not affect the long-term duration of $\left[\mathrm{Ca}^{2+}\right]_{i}$ oscillations. $\left[\mathrm{Ca}^{2+}\right]_{i}$ profiles induced by ICSI in eggs from which a comparable amount ooplasm was removed $(A$ and $D)$, or from eggs in which the sperm head was removed (B and $E$ ), or in non-manipulated fertilized eggs (C). Following ICSI, eggs were cultured for $60 \mathrm{~min}$ in KSOM in the presence of colcemid $(100 \mathrm{ng} / \mathrm{ml})$ prior to enucleation. Following enucleation and during $\left[\mathrm{Ca}^{2+}\right]_{i}$ monitoring, zygotes were maintained in the presence of colcemid. A break in the $X$-axis reflects time in culture without monitoring to decrease detrimental effects of extended u.v.-light exposure. The scheme representing the time line for the each of the procedures performed to complete the experiment is shown above the $\left[\mathrm{Ca}^{2+}\right]_{i}$ panels. 
enucleated zygotes were capable of mounting a $\left[\mathrm{Ca}^{2+}\right]_{\mathrm{i}}$ rise associated with PNBD. To shorten the time to PNBD, we made use of OA, a phosphatase inhibitor that has been shown to induce premature PNBD (Dyban et al. 1993, Moos et al. 1995), and that we have demonstrated causes a PNBD-associated $\left[\mathrm{Ca}^{2+}\right]_{\mathrm{i}}$ rise in fertilized zygotes (Gordo et al. 2002). As shown in Fig. $3 \mathrm{~A}$ and $\mathrm{B}, \mathrm{OA}$ induced $\mathrm{a}\left[\mathrm{Ca}^{2+}\right]_{i}$ rise associated with PNBD in 18/18 fertilized zygotes and in 8/10 zygotes in which a part of the ooplasm was removed. OA treatment also evoked a $\left[\mathrm{Ca}^{2+}\right]_{i}$ rise associated with PNBD in the majority of enucleated zygotes (6/10; Fig. 3C, $P>0.05$ ), whereas it failed to do so in control, $\mathrm{SrCl}_{2}$-activated zygotes $(0 / 11$; Fig. 3D). It is worth noting that by the time that the PNBD-associated $\left[\mathrm{Ca}^{2+}\right]_{i}$ rise was detected, the nuclear envelope in all these zygotes was no longer visible, although nucleoli remnants could still be observed in a few cases. Collectively, the results suggest that in enucleated zygotes the SF achieves the correct cellular distribution.

\section{Loss of PLCל coincides with loss of sperm's ability to induce $\left[\mathrm{Ca}^{2+}\right]_{i}$ oscillations}

The sperm-specific PLC $\zeta$ represents the strongest candidate to date to be the sperm's SF (Saunders et al. 2002, Larman et al. 2004, Kurokawa et al. 2005, Kuroda et al. 2006). However, the localization of PLC $\zeta$ in sperm, its temporal release and, ultimately, whether its absence precludes the initiation of oscillations at fertilization has not been demonstrated. To examine PLC $\zeta$ localization in mouse and bovine sperm, we made use two of different anti $(\alpha)$-PLC $\zeta$ antibodies raised by our laboratory (Kurokawa et al. 2005). The specificity of these antibodies was characterized in a previous manuscript (Kurokawa et al. 2005) and they were shown, using Western blotting, to recognize a band of $\sim 74 \mathrm{kDa}$ relative molecular weight $(\mathrm{rMW})$ in mouse sperm ( $\alpha \mathrm{PLC} \zeta-\mathrm{CT})$, which is the expected

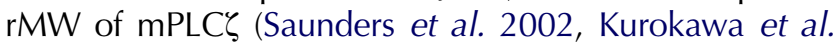
2005), and a band of $\sim 72 \mathrm{kDa}$ in bull sperm, which is consistent with the expected rMW of bPLC $\zeta$ (Malcuit et al. 2005). We therefore next investigated by immunofluorescence the localization of PLC $\zeta$ in mouse and bull sperm. As expected, sperm stained only with the secondary antibody, which served as negative controls, showed absence of reactivity (Fig. 4A and E). Importantly, addition of either primary antibody revealed in mouse sperm a distinct postacrosomal band, approximately at the post-equatorial region of the sperm head (Fig. 4C and D), which is in agreement with the only previous study that examined

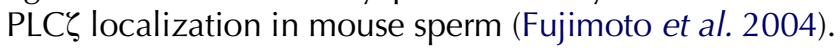
Our antibodies also labeled the sperm tail and the acrosome region (Fig. 4B-D; inset in $4 \mathrm{~B}$ shows PNA staining), although the antigenic peptide for the NT antibody only obliterated the equatorial staining (Fig. 4B), which suggest that the post-acrosomal labeling
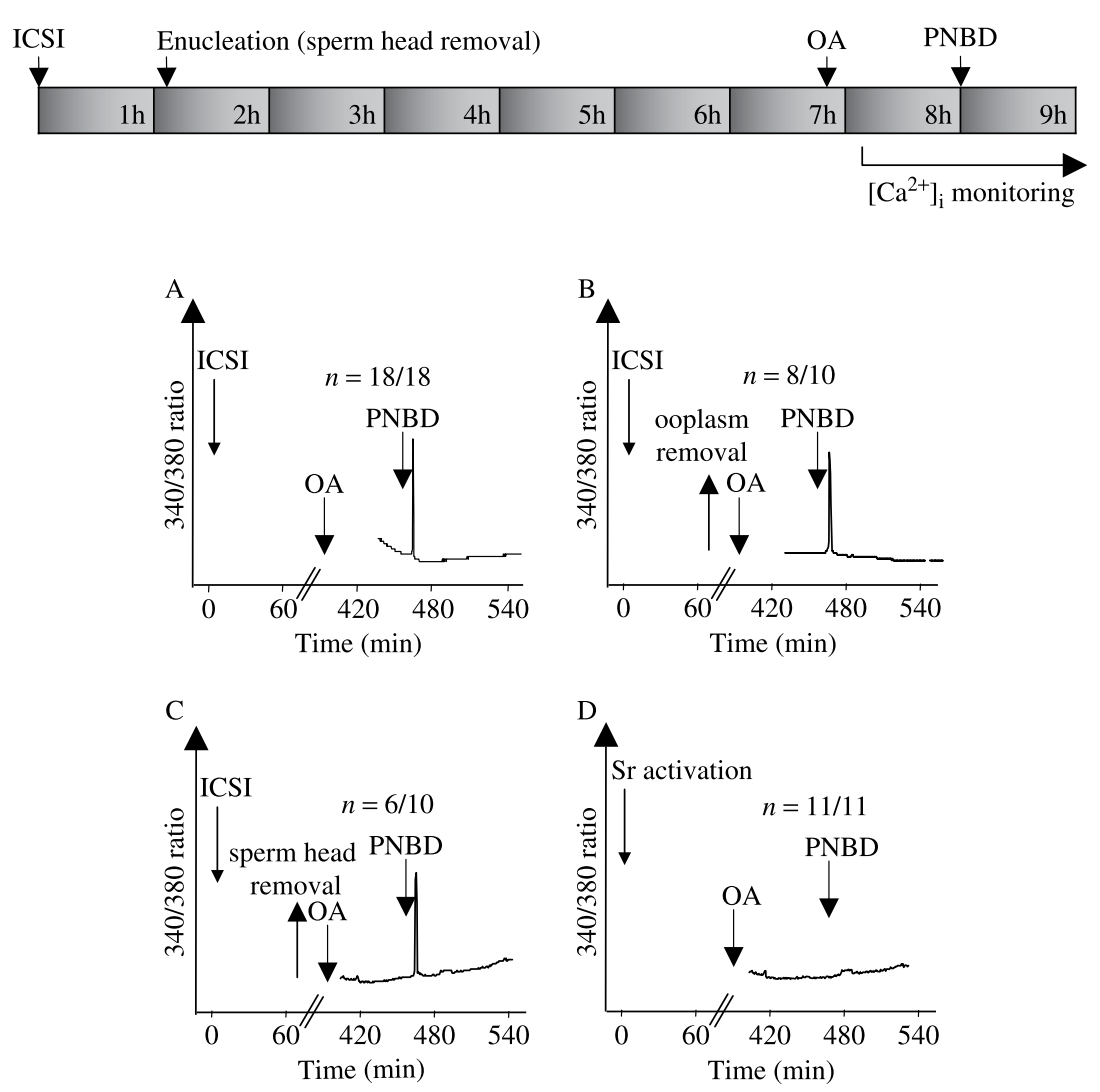

Figure 3 Sperm enucleation after ICSI does not affect the association of the SF with the PN. Premature PNBD was induced by the addition of $10 \mu \mathrm{MOA} . \mathrm{A}\left[\mathrm{Ca}^{2+}\right]_{i}$ rise was observed in controlICSI fertilized eggs (A), in eggs in which ooplasm was removed (B), and in enucleated eggs (C). A $\left[\mathrm{Ca}^{2+}\right]_{i}$ rise was not induced in $\mathrm{SrCl}_{2-}$ activated eggs (D). The scheme representing the time line for each of the procedures performed to complete the experiment is shown above the $\left[\mathrm{Ca}^{2+}\right]_{i}$ panels. 

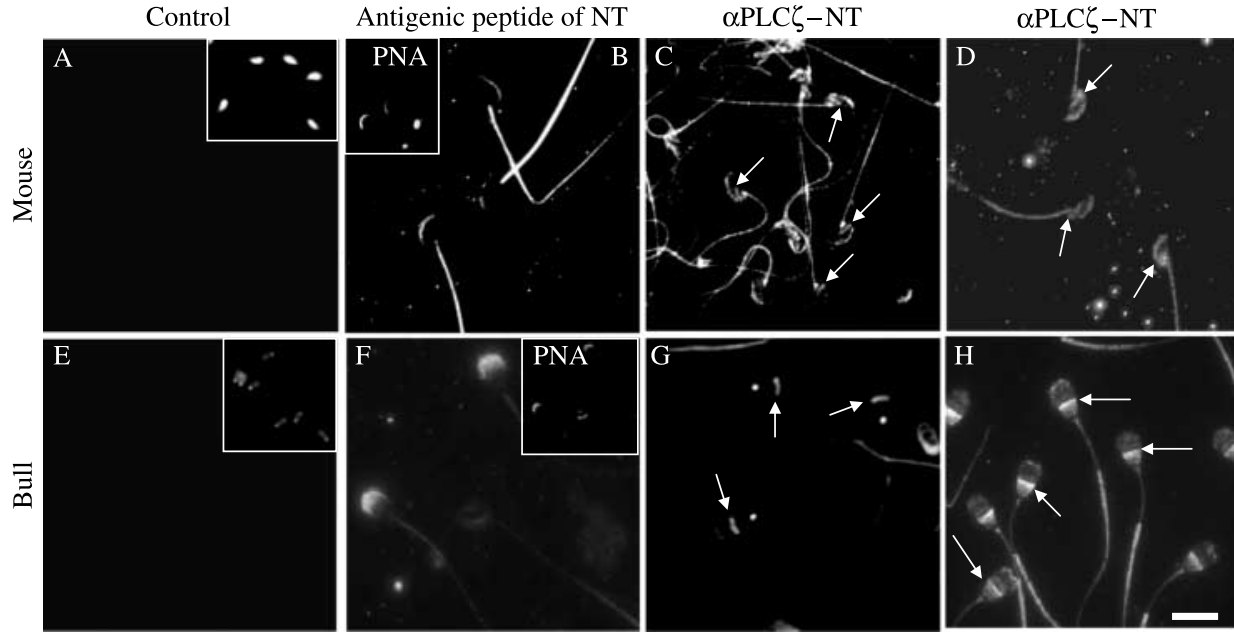

Figure 4 PLC $\zeta$ is localized to the post-acrosomal region of mouse sperm (A-D) and to the equatorial region of bull sperm (E-H). Fresh mouse and bull sperm reactivity in the absence of primary antibody ( $\mathrm{A}$ and $\mathrm{E}$ ); insets show sperm nuclei stained with Hoechst 33342 . In the presence of antigenic peptide (30 $\mu \mathrm{g}$ peptide per $\mu \mathrm{l}$ of anti-serum of $\alpha \mathrm{PLC} \zeta-\mathrm{NT})$, a fluorescent signal was detectable in the acrosome and tail suggesting that the signal at these locations was nonspecific and due to cross-reactivity (B and F); insets show acrosomes stained with PNA-lectin (PNA). Mouse and bull sperm

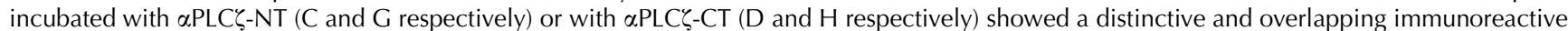
signal (arrow) at the post-acrosomal region (mouse) and equatorial region (bull). Scale bar in $G=10 \mu \mathrm{m}$.

specifically denotes the localization of PLCל. In bull sperm, the antibodies also generated overlapping immunostaining patterns, with strong reactivity detected in the post acrosomal, equatorial region of the sperm head (Fig. 4G and H). As was the case for mouse sperm, non-specific fluorescence was observed to the acrosomal and tail regions (Fig. 4G and $\mathrm{H}$ ), as it was not alleviated by pre-incubation of the antibody with its corresponding antigenic peptide (Fig. 4F, inset (PNA) shows acrosomal region). Thus, given that in sperm of both species, the addition of the NT antigenic peptide exclusively prevented the post-acrosomal (mouse) and equatorial (bull) signals, and that the antibodies provided overlapping fluorescent signals in both species, we can therefore conclude that the described localization represents the correct PLÇ distribution for these species.

To ascertain whether the presence of PLC $\zeta$ corresponds with the ability of sperm to initiate oscillations, we first examined whether mouse and bull sperm exposed to a brief treatment with high $\mathrm{pH}$, a procedure that we have shown uncouples the SF activity from the sperm nuclei (Kurokawa et al. 2005), were able to
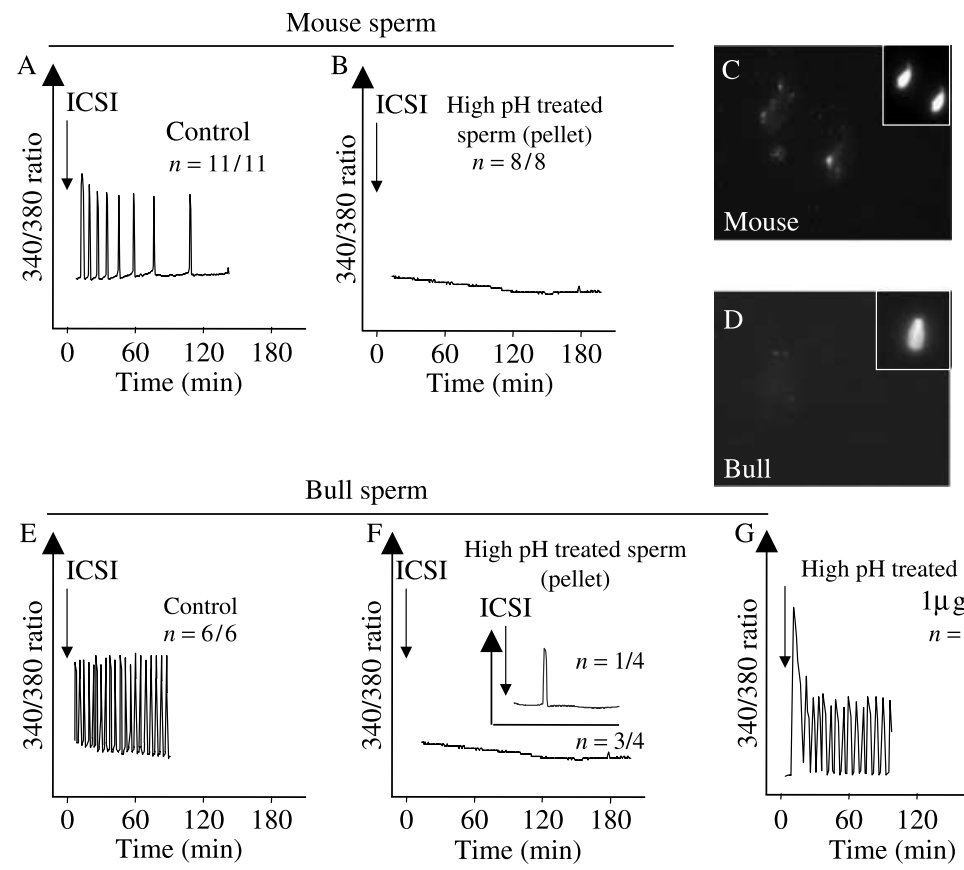

Bull sperm

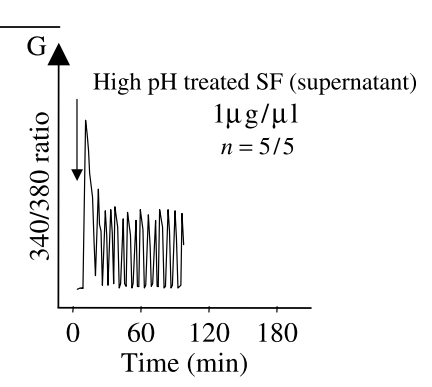

Figure 5 High-pH treatment abolishes SF activity and abrogates PLC $\zeta$ immunoreactivity in mouse $(A-C)$ and bull sperm (D-G). Injection of a mouse (A) or a bull sperm (E) into mouse eggs initiates $\left[\mathrm{Ca}^{2+}\right]_{\mathrm{i}}$ responses and this activity is depleted by treating the sperm with high $\mathrm{pH}$ (B and $\mathrm{F}$ respectively). Importantly, $\mathrm{pH}$ treatment also obliterated PLC $\zeta$ immunoreactivity from sperm of both species ( $\alpha$ PLC $\zeta-N T, C$ and D). Insets in $C$ and $D$ show sperm nuclear staining with Hoechst 33258. 


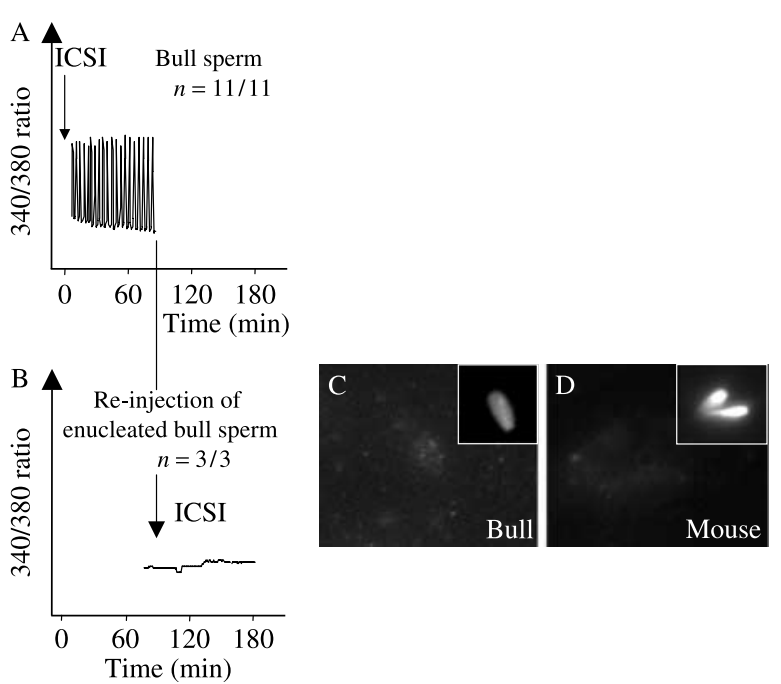

Figure 6 Loss of SF activity coincides with the loss of sperm PLC immunoreactivity after enucleation. $\left[\mathrm{Ca}^{2+}\right]_{i}$ profile of an egg after injection of a fresh bull sperm (A), or of an egg after injection with the same bull sperm enucleated 90 min after ICSI (B). Residence in the ooplasm also depleted PLC $\zeta$ immunoreactivity from bull (C) and mouse sperm (D); insets show the presence of bull and mouse sperm nuclei in the slides (Hoechst 33 258).

initiate oscillations upon injection into mouse eggs. High-pH treatment abrogated the $\left[\mathrm{Ca}^{2+}\right]_{i}$ oscillatory activity of all mouse ( $n=8 / 8$; Fig. $5 \mathrm{~A}$ and $\mathrm{B}$ ) and bull sperm ( $n=6 / 6$; Fig. 5E and F) and, more importantly, it eliminated PLC $\zeta$ immunoreactivity from sperm of both species (Fig. 5C and D respectively; insets show Hoechst-stained sperm heads). Importantly, the supernatants recovered from these sperm exhibited undisturbed $\left[\mathrm{Ca}^{2+}\right]_{i}$ oscillatory activity when injected in mouse MII eggs (Fig. 5G), demonstrating that the $\mathrm{pH}$ treatment did not eliminate PLC $\zeta$ immunoreactivity by inactivating the enzyme. In addition, PNA staining of both mouse and bull sperm after high-pH treatment resulted in the presence of specific staining (data not shown) that has been reported to develop after sperm undergo the acrosome reaction (Cheng et al. 1996, Baker et al. 2004), suggesting that not all sperm head-associated proteins were removed by the high-pH treatment.

We next investigated whether the temporal release of PLC $\zeta$ from the sperm head concurred with the previously noted release of SF activity (Knott et al. 2003), and whether loss of PLC $\zeta$ immunoreactivity prevented the ability of sperm to induce $\left[\mathrm{Ca}^{2+}\right]_{i}$ oscillations. To examine these questions, we first performed ICSI followed by sperm head enucleation within $90 \mathrm{~min}$ post-ICSI. The aspirated sperm heads were either stained for PLC $\zeta$ reactivity or re-injected into MII eggs. As expected, re-injection of enucleated bull or mouse sperm heads failed to induce oscillations in mouse eggs (Fig. 6B). Importantly, all enucleated sperm heads examined lacked PLC $\zeta$ immunoreactivity (Fig. 6C and D). It is worth noting that while mouse sperm heads had started to undergo decondensation by this time (Fig. 6D inset), this was not the case for bull sperm heads (Fig. 6C inset), suggesting that, at least for the latter, the loss of PLC $\zeta$ could not be attributed to massive exodus of sperm proteins into the ooplasm. Together, the results show that mouse and bull sperm depleted of $\mathrm{PLC} \zeta$ are incapable of inducing $\left[\mathrm{Ca}^{2+}\right]_{\mathrm{i}}$ oscillations in eggs.

\section{Discussion}

The present study investigated whether the SF activity of mouse and bovine sperm and their PLC $\zeta$ immunoreactivity were fully released into the ooplasm within the first 90 min post-fertilization. We also examined whether the loss of both of these properties impacted the ability of these sperm to initiate $\left[\mathrm{Ca}^{2+}\right]_{i}$ oscillations. We found, consistent with our previously published results (Knott et al. 2003), that indeed all the SF activity, i.e. the ability of the sperm to induce persistent oscillations, was released into the ooplasm within the first $2 \mathrm{~h}$ of sperm entry. However, in the previous manuscript, we only examined the persistence of the oscillations until the PN stage, a stage at which in mouse eggs $\left[\mathrm{Ca}^{2+}\right]_{i}$ oscillations cease spontaneously (Jones et al. 1995, Day et al. 2000, Deguchi et al. 2000, Marangos et al. 2003, Lee et al. 2006). Therefore, here, we extended the oscillatory permissive state of eggs by arresting zygotes at a MII-like stage and appropriately prolonging the $\left[\mathrm{Ca}^{2+}\right]_{i}$ monitoring period. We found that enucleated zygotes were able to sustain oscillations as long as those of fertilized and non-manipulated zygotes under any of the conditions tested. Therefore, our findings together with those in the literature allow us to propose a model of SF release during mouse fertilization whereby the SF starts to trickle from the sperm into the ooplasm soon after the fusion of the gametes (Lawrence et al. 1997, Jones et al. 1998b) and this continues uninterrupted for the next 60 to $70 \mathrm{~min}$, at which time the totality of the SF activity has been deposited in the ooplasm (this study and Knott et al. 2003). This protracted release of the SF may be due, at least in part, to the time required to achieve full disassembly of the PT, the sperm domain thought to host the SF activity (Kimura et al. 1998, Perry et al. 1999, Knott et al. 2003). From a functional standpoint, the slow release of the $\left[\mathrm{Ca}^{2+}\right]_{i}$ oscillation-inducing factor may ensure continued availability of the SF for the first few hours following fertilization, such that the pattern of $\left[\mathrm{Ca}^{2+}\right]_{i}$ oscillations exhibits a steady pace until the progression into interphase is eminent, as signaled by the impending $\mathrm{PN}$ formation.

Once released into the ooplasm, the SF seemingly distributes throughout the ooplasm making possible the generation of oscillations and then, as zygotes progress into interphase, it becomes associated with the PN, as evidenced by the results showing that injection of these PNs into fresh MII eggs initiates $\left[\mathrm{Ca}^{2+}\right]_{i}$ oscillations (Kono et al. 1996, Ogonuki et al. 2001, Knott et al. 
2003). This SF association with the PN was further corroborated by the findings that inhibition of either PN formation using wheat germ agglutinin or abrogation of molecular transport into the PN abrogated the interphase-linked termination of the $\left[\mathrm{Ca}^{2+}\right]_{i}$ oscillations (Marangos et al. 2003). Our results show that the released SF attains the expected cellular distribution even in the absence of the fertilizing sperm head, as fertilized/enucleated zygotes and non-manipulated fertilized zygotes exhibited the $\left[\mathrm{Ca}^{2+}\right]_{i}$ rise associated with PNBD (Jellerette et al. 2004, Larman et al. 2004), which here was prematurely induced by exposure to OA. The association/incorporation of the SF activity with the $\mathrm{PN}$ is consistent with the recent demonstration that the linker region of putative $\mathrm{SF}, \mathrm{PLC} \zeta$, contains a positively charged consensus nuclear localization signal (Saunders et al. 2002, Larman et al. 2004). It is presently unclear why the SF activity/PLC $\zeta$ becomes incorporated into the $\mathrm{PN}$, although it might perform targeted signaling in the $\mathrm{PN}$ by inducing persistent stimulation of the PI pathway (Sone et al. 2005). Furthermore, it cannot be discounted that given the developmental risks of excessive/abnormal stimulation of the PI pathway/[Ca $\left.{ }^{2+}\right]_{i}$ oscillations (Gordo et al. 2000, Rogers et al. 2004, Ozil et al. 2005), the PN sequestration of PLÇ may act as an insurance mechanism to prevent the detrimental effects of persistent $\left[\mathrm{Ca}^{2+}\right]_{\mathrm{i}}$ oscillations (Marangos et al. 2003, Larman et al. 2004).

Since its discovery by Saunders et al. (2002), accumulating research has solidified the view that PLC $\zeta$ is likely to represent the SF in all mammals (see also Introduction, Cox et al. 2002, Saunders et al. 2002, Kurokawa et al. 2005, Yoneda et al. 2006). Nevertheless, many features of this molecule and its function remain to be elucidated. Among the least investigated characteristics of PLC $\zeta$ is its localization in sperm. The SF activity has been shown to be associated with the PT of the sperm head (Perry et al. 2000, Knott etal. 2003) and, consistent with those findings, the only report that examined the localization of PLC $\zeta$ found that it distributes to the post-acrosomal region of mouse sperm (Fujimoto et al. 2004). This is an appropriate distribution for the putative $\left[\mathrm{Ca}^{2+}\right]_{i}$ oscillation-inducing factor, since reportedly the contents of this region quickly mingle with the ooplasm (Manandhar \& Toshimori 2003, Sutovsky et al. 2003). In the present study, we extend those results by examining the localization of $\mathrm{PLC} \zeta$ in mouse and bull sperm with antibodies raised against peptide sequences from opposite ends of the molecule (Kurokawa et al. 2005). We selected sperm from these species as they show greatly different sperm shapes, with the round shape of bull sperm more reminiscent of the sperm shape of humans and other large mammals. Our immunofluorescence results confirm the post-acrosomal localization of PLC $\zeta$ in mouse sperm and, for the first time, show that in bull sperm PLC $\zeta$ is distributed to the equatorial region, a region that is also expected to gain rapid access to the ooplasm (Sutovsky et al. 2003). It is important to note that our localization studies were performed in non-capacitated and non-acrosome-reacted sperm, changes that may alter the localization/conformation of PLC $\zeta$ to favor its release/activation. Thus, PLC $\zeta$ localization studies should also be performed in future studies in capacitated and acrosome-reacted sperm. Lastly, it is worth noting that the presence of a $53 \mathrm{kDa}$ immunoreactive polypeptide was reported in extracts from sperm tails using an anti-PLC $\zeta$ antibody (Fujimoto et al. 2004), and detection of this polypeptide by our antibodies may account for some of the non-specific staining observed in our preparations. Nonetheless, it is unlikely that this protein represents a fragment of PLC $\zeta$ as this reactivity was not abolished by the corresponding antigenic peptide.

Whether or not the loss of PLC $\zeta$ affects the ability of sperm to initiate oscillations remains untested since a knockout mouse is not yet available. Moreover, the temporal release of PLC $\zeta$ during fertilization is also an outstanding question. To start answering these questions, we depleted the content of PLC $\zeta$ from sperm using a high-pH treatment, which we have shown releases all the SF activity from the sperm into the supernatant (Kurokawa et al. 2005). PLC $\zeta$ was also depleted in a more physiological manner by enucleating the fertilizing sperm from the ooplasm after a time that is known to deplete the sperm's oscillatory activity (Knott et al. 2003). Under both conditions and in sperm of both species, the loss of the sperm's $\left[\mathrm{Ca}^{2+}\right]_{i}$ oscillatory activity coincided with the loss of PLC $\zeta$ immunoreactivity, which occurred by approximately $90 \mathrm{~min}$ post-fertilization. Together, these results strengthen the view that PLC $\zeta$ is the putative factor responsible for the initiation of the $\left[\mathrm{Ca}^{2+}\right]_{i}$ oscillations at fertilization in mammals. Future studies should examine the distribution of PLC $\zeta$ within the ooplasm soon after fertilization. We were unable to perform these studies given the high cross-reactivity of our antibodies with egg proteins.

In summary, our results demonstrate that both the SF activity and the PLCY content are emptied into the ooplasm within $90 \mathrm{~min}$ of sperm entry and that absence of PLCל reactivity coincides with loss of $\left[\mathrm{Ca}^{2+}\right]_{i}$ oscillatory activity of mammalian sperm. These results support the notion that PLC $\zeta$ is necessary and sufficient to induce egg activation in mammals.

\section{Materials and Methods}

\section{Gamete collection}

Metaphase II arrested eggs were recovered from B6D2F1 $(\mathrm{C} 57 \mathrm{BL} / 6 \mathrm{~J} \times \mathrm{DBA} / 2 \mathrm{~J})$ female mice (6-8 weeks old) that were induced to ovulate by injection of $5 \mathrm{IU}$ of human chorionic gonadotropin (hCG; Sigma), which was administered 46-48 h after an injection of $5 \mathrm{IU}$ pregnant mare serum gonadotropin (Sigma). Eggs were collected from the oviducts $14-15 \mathrm{~h}$ posthCG in HEPES-buffered tyrode-lactate solution (TL-HEPES) supplemented with $5 \%$ heat-treated fetal calf serum (FCS; 
Gibco, BRL). Cumulus cells were removed by a brief treatment with $0.1 \%$ bovine testes hyaluronidase (Sigma). Mouse sperm were collected from the cauda epididymides of B6D2F1 or CD1 male mice (11-to 24-weeks old) and washed two times with injection buffer (IB, $75 \mathrm{mM} \mathrm{KCl}$ and $20 \mathrm{mMHEPES}, \mathrm{pH}$ 7.0). Bull sperm were obtained from frozen semen samples (kindly donated by Dr Marvin Pace, American Breeder Services, DeForest, WI, USA) following selection using Percoll gradients (90-45\%) according to the procedure described by others (Parrish et al. 1985). The selected live and motile sperm were washed twice and then resuspended in a Dulbeco's PBS (DPBS). Bull sperm were decapitated by a $30 \mathrm{~s}$ sonication on ice (XL2020; Heat Systems, Farmingdale, NY, USA). High-pH treatment was used to deplete the $\left[\mathrm{Ca}^{2+}\right]_{i}$ oscillation-inducing activity from mouse and bull sperm using a procedure previously described by our laboratory (Kurokawa et al. 2005). In brief, after sonication and centrifugation, sperm pellets were washed with sperm buffer $(75 \mathrm{mM} \mathrm{KCl}, 20 \mathrm{mM}$ HEPES, $1 \mathrm{mM}$ EGTA, $10 \mathrm{mM}$ glycerophosphate, $1 \mathrm{mM}$ dithiothreitol, $200 \mu \mathrm{M}$ phenylmethylsulphonyl fluoride, $10 \mathrm{mg} / \mathrm{ml}$ pepstatin, $10 \mathrm{mg} / \mathrm{ml}$ leupeptin, $\mathrm{pH}$ 7.0), followed by a 30-min incubation in a high salt solution ( $1 \mathrm{M} \mathrm{KCl}, 10 \mathrm{mM}$ Tris, $\mathrm{pH} \mathrm{7.4)}$ at $4{ }^{\circ} \mathrm{C}$ after which sperm were exposed to an alkaline carbonate solution $(100 \mathrm{mM}$ $\mathrm{Na}_{2} \mathrm{CO}_{3}, \mathrm{pH} 11.5$ ) for 10 min at $4{ }^{\circ} \mathrm{C}$. The sperm suspension was then neutralized with $0.5 \mathrm{M}$ Tris $(\mathrm{pH} 3.0)$ and washed with DPBS. All procedures were performed according to standard animal protocols approved by the University of Massachusetts Animal Care Committee.

\section{Intracytoplasmic sperm injection (ICSI) and sperm enucleation}

ICSI was performed as previously described (Kimura \& Yanagimachi 1995, Kurokawa \& Fissore 2003) using Narishige manipulators (Medical Systems Corp., Great Neck, NY, USA) mounted on a Nikon Diaphot microscope (Nikon Inc., Garden City, NY, USA). All manipulations were carried out in $50 \mu \mathrm{l}$ drops of HEPES-buffered CZB media (Chatot et al. 1989) under light mineral oil at room temperature (RT). Sperm were washed in IB and mixed with one part IB containing $12 \%$ polyvinyl pyrrolidone (MW 360 kDa; Sigma). Single sperm was aspirated into a bluntended pipette driven by a Piezo electric unit (Burleigh, Rochester, NY, USA). Several Piezo pulses were applied to separate the head from the tail and different intensity pulses were used to penetrate the zona pellucida and plasma membrane. After ICSI, zygotes were cultured in potassium simplex optimized medium (Specialty Media, Lavallette, NJ, USA) for $90 \mathrm{~min}$. Enucleation, a term here exclusively reserved to indicate removal of the sperm head from the ooplasm, was carried out using the same set up and as previously described by our laboratory (Knott et al. 2003). Prior to enucleation, eggs were incubated in $3 \mu \mathrm{g} / \mathrm{ml}$ Hoechst 33342 for $5 \mathrm{~min}$ at RT. Enucleation was accomplished by bringing the pipette near the Hoechst-stained sperm head, the precise location of which was established by brief pulses of u.v. light, followed by aspiration using an IM-55-2 Narishige syringe. Control eggs underwent the same manipulation procedure, but a comparable volume of ooplasm was aspirated. Enucleated sperm heads used for immunostaining were freed of the surrounding cytoplasm by administration of Piezo pulses.

\section{$\left[\mathrm{Ca}^{2+}\right]_{i}$ monitoring}

$\left[\mathrm{Ca}^{2+}\right]_{\mathrm{i}}$ monitoring was carried out as previously described (Jellerette et al. 2004). Eggs were first loaded with $1 \mu \mathrm{M}$ Fura 2-acetoxymethyl ester (Molecular Probes, Eugene, OR, USA) supplemented with $0.02 \%$ pluronic acid (Molecular Probes) for 20 min at RT and then transferred into $50 \mu \mathrm{l}$ drops of TL-HEPES (without FCS) placed on a glass coverslip sealed over an opening in the bottom of a culture dish and covered with mineral oil. Eggs were monitored simultaneously using a $20 \times$ objective on a Nikon Diaphot inverted microscope (Nikon Corp., Tokyo, Japan) fitted for fluorescence measurements. A $75 \mathrm{~W}$ Xenon lamp provided the excitation light. The excitation wavelength was alternated between 340 and $380 \mathrm{~nm}$ by a filter wheel (Ludl Electronic Products, Hawthorne, NY, USA) and fluorescence ratios were obtained every 20 or $30 \mathrm{~s}$. The emitted light was passed through a $510 \mathrm{~nm}$ barrier filter and collected with either a cooled Photometrics SenSys CCD or a cool SNAP ES digital camera (Roper Scientific, Tucson, AZ, USA). SimplePCI software (Compix Imaging Inc., Cranberry, PA, USA) was used to monitor $\left[\mathrm{Ca}^{2+}\right]_{i}$ and synchronize the rotation of the filter wheel. $\left[\mathrm{Ca}^{2+}\right]_{\mathrm{i}}$ values are reported as the ratio of $340 / 380 \mathrm{~nm}$ fluorescence in the whole egg.

\section{Antibodies and other chemicals}

Two different anti-PLC $\zeta$ rabbit sera were raised (Kurokawa et al. 2005): one against a 19-mer sequence (GYRRVPLFSKSGANLEPSS) on the C-terminus of mouse (m) PLC $\zeta$ (accession no. NP_473403; Saunders et al. 2002) and the other against a 19-mer sequence (MENKWFLSMVRDDFKGGKI) on the N-terminus of pig (p) PLC $\zeta$ (accession no. BAC78817; Kurokawa et al. 2005). The antibodies specifically recognized PLC $\zeta$ in mouse and porcine sperm in Western blots respectively (Kurokawa et al. 2005). Okadaic acid (OA; Sigma), a phosphatase inhibitor, was dissolved in TL-HEPES medium and used at $10 \mu \mathrm{M}$ as a final concentration.

\section{Sperm immunofluorescence}

Sperm were fixed in $3.7 \%$ paraformaldehyde for 30 min at $4{ }^{\circ} \mathrm{C}$ followed by permeabilization with $0.1 \%(\mathrm{v} / \mathrm{v})$ Triton X-100DPBS for $10 \mathrm{~min}$ at RT. The sperm suspension was then spotted as $50 \mu \mathrm{l}$ drops onto $0.1 \%$ poly L-lysine pre-coated glass slides (Erie Sci., Portsmouth, NH, USA) and allowed to attach to the slide for $20 \mathrm{~min}$ at $37^{\circ} \mathrm{C}$. Sperm were incubated in $5 \%$ normal goat serum (NGS) in DPBS for $3 \mathrm{~h}$ at $4{ }^{\circ} \mathrm{C}$ and then incubated overnight at $4{ }^{\circ} \mathrm{C}$ with anti-pPLC $\zeta(\mathrm{NT} ; 1: 100)$ or anti-mPLC $\zeta(\mathrm{CT}$; $1: 100)$ in $5 \%$ NGS. After several washes with $0.1 \%(\mathrm{v} / \mathrm{v})$ Tween 20-DPBS (DPBS-T), a secondary, Alexa Fluor 555-labeled goat anti-rabbit antibody $(1: 200)$ was added for $1 \mathrm{~h}$ at RT. Detection of the acrosome was performed by incubating the same sperm samples with $20 \mu \mathrm{g} / \mathrm{ml}$ Alexa Fluor 488-conjugated peanut agglutinin (PNA)-lectin (from Arachis hypogaea peanut; Molecular Probes) in 5\% NGS for $30 \mathrm{~min}$ at RT. After several washings in DPBS-T, samples were counterstained with $5 \mu \mathrm{g} / \mathrm{ml}$ Hoechst 33258 and mounted using the Vectashield mounting media (Vector Laboratories, Burlingame, CA, USA). Fluorescence images were obtained using a Zeiss Axiovert $200 \mathrm{M}$ 
microscope with a $63 \times$ oil immersion objective and a Hamamatsu Orca AG-cooled CCD Camera under the control of Openlab software (Improvision, Lexington, MA, USA).

\section{Statistical analysis}

Comparisons of $\left[\mathrm{Ca}^{2+}\right]_{i}$ parameters were performed using Student's t-test. All experiments were repeated at least three times. Statistical comparisons were carried out using the Sigmaplot software (SPW 8.0, SPSS Inc., Chicago, IL, USA). Significance was set at $P<0.05$.

\section{Acknowledgements}

This work was supported in part by grants from the National Research Initiative Competitive Grants Program from the USDA (2007-35203-17840), a Cooperative State Research, Education, and Extension Service grant (Hatch/USDA) and by an RO3 grant from the NIH to R A F. We thank Drs Manabu Kurokawa and Bora Lee for reading the manuscript and helpful suggestions. We acknowledge the technical assistance of Ms Changli He. The authors declare that there is no conflict of interest that would prejudice the impartiality of this scientific work.

\section{References}

Baker SS, Thomas M \& Thaler CD 2004 Sperm membrane dynamics assessed by changes in lectin fluorescence before and after capacitation. Journal of Andrology 25 744-751.

Berrie CP, Cuthbertson KS, Parrington J, Lai FA \& Swann K 1996 A cytosolic sperm factor triggers calcium oscillations in rat hepatocytes. Biochemical Journal 313 369-372.

Chatot CL, Ziomek CA, Bavister BD, Lewis JL \& Torres I 1989 An improved culture medium supports development of random-bred 1-cell mouse embryos in vitro. Journal of Reproduction and Fertility 86 679-688.

Cheng FP, Fazeli A, Voorhout WF, Marks A, Bevers MM \& Colenbrander B 1996 Use of peanut agglutinin to assess the acrosomal status and the zona pellucida-induced acrosome reaction in stallion spermatozoa. Journal of Andrology 17 674-682.

Cox LJ, Larman MG, Saunders CM, Hashimoto K, Swann K \& Lai FA 2002 Sperm phospholipase Czeta from humans and cynomolgus monkeys triggers $\mathrm{Ca}^{2+}$ oscillations, activation and development of mouse oocytes. Reproduction 124 611-623.

Day ML, McGuinness OM, Berridge MJ \& Johnson MH 2000 Regulation of fertilization-induced $\mathrm{Ca}^{2+}$ spiking in the mouse zygote. Cell Calcium 28 47-54.

Deguchi R, Shirakawa H, Oda S, Mohri T \& Miyazaki S 2000 Spatiotemporal analysis of $\mathrm{Ca}^{2+}$ waves in relation to the sperm entry site and animal-vegetal axis during $\mathrm{Ca}^{2+}$ oscillations in fertilized mouse eggs. Developmental Biology 218 299-313.

Dong JB, Tang TS \& Sun FZ 2000 Xenopus and chicken sperm contain a cytosolic soluble protein factor which can trigger calcium oscillations in mouse eggs. Biochemical and Biophysical Research Communications 268 947-951.

Ducibella T, Huneau D, Angelichio E, Xu Z, Schultz RM, Kopf GS, Fissore R, Madoux S \& Ozil JP 2002 Egg-to-embryo transition is driven by differential responses to $\mathrm{Ca}^{2+}$ oscillation number. Developmental Biology 250 280-291.

Dupont G, McGuinness OM, Johnson MH, Berridge MJ \& Borgese F 1996 Phospholipase $\mathrm{C}$ in mouse oocytes: characterization of beta and gamma isoforms and their possible involvement in sperm-induced $\mathrm{Ca}^{2+}$ spiking. Biochemical Journal 316 583-591.

Dyban AP, De Sutter P \& Verlinsky Y 1993 Okadaic acid induces premature chromosome condensation reflecting the cell cycle progression in onecell stage mouse embryos. Molecular Reproduction and Development 34 402-415.
FitzHarris G, Marangos P \& Carroll J 2003 Cell cycle-dependent regulation of structure of endoplasmic reticulum and inositol 1,4,5-trisphosphateinduced $\mathrm{Ca}^{2+}$ release in mouse oocytes and embryos. Molecular Biology of the Cell 14 288-301.

Fujimoto S, Yoshida N, Fukui T, Amanai M, Isobe T, Itagaki C, Izumi T \& Perry AC 2004 Mammalian phospholipase Czeta induces oocyte activation from the sperm perinuclear matrix. Developmental Biology 274 370-383

Gordo AC, Wu H, He C \& Fissore RA 2000 Injection of sperm cytosolic factor into mouse metaphase II oocytes induces different developmental fates according to the frequency of $\left[\mathrm{Ca}^{2+}\right]$ i oscillations and oocyte age. Biology of Reproduction 62 1370-1379.

Gordo AC, Kurokawa M, Wu H \& Fissore RA 2002 Modifications of the $\mathrm{Ca}^{2+}$ release mechanisms of mouse oocytes by fertilization and by sperm factor. Molecular Human Reproduction 8 619-629.

Jellerette T, Kurokawa M, Lee B, Malcuit C, Yoon S-Y, Smyth J, Vermassen E, De Smedt H, Parys JB \& Fissore RA 2004 Cell cycle-coupled $\left[\mathrm{Ca}^{2+}\right] \mathrm{i}$ oscillations in mouse zygotes and function of the inositol 1,4,5trisphosphate receptor-1. Developmental Biology 274 94-109.

Jones KT, Carroll J, Merriman JA, Whittingham DG \& Kono T 1995 Repetitive sperm-induced $\mathrm{Ca}^{2+}$ transients in mouse oocytes are cell cycle dependent. Development 121 3259-3266.

Jones KT, Cruttwell C, Parrington J \& Swann K 1998a A mammalian sperm cytosolic phospholipase $\mathrm{C}$ activity generates inositol trisphosphate and causes $\mathrm{Ca}^{2+}$ release in sea urchin egg homogenates. FEBS Letters 437 297-300.

Jones KT, Soeller C \& Cannell MB $1998 b$ The passage of $\mathrm{Ca}^{2+}$ and fluorescent markers between the sperm and egg after fusion in the mouse. Development 125 4627-4635.

Kimura Y \& Yanagimachi R 1995 Intracytoplasmic sperm injection in the mouse. Biology of Reproduction 52 709-720.

Kimura Y, Yanagimachi R, Kuretake S, Bortkiewicz H, Perry AC \& Yanagimachi H 1998 Analysis of mouse oocyte activation suggests the involvement of sperm perinuclear material. Biology of Reproduction $\mathbf{5 8}$ 1407-1415.

Knott JG, Kurokawa M \& Fissore RA 2003 Release of the $\mathrm{Ca}^{2+}$ oscillationinducing sperm factor during mouse fertilization. Developmental Biology 260 536-547.

Knott JG, Kurokawa M, Fissore RA, Schultz RM \& Williams CJ 2005 Transgenic RNA interference reveals role for mouse sperm phospholipase Czeta in triggering $\mathrm{Ca}^{2+}$ oscillations during fertilization. Biology of Reproduction 72 992-996.

Kono T, Jones KT, Bos-Mikich A, Whittingham DG \& Carroll J 1996 A cell cycle-associated change in $\mathrm{Ca}^{2+}$ releasing activity leads to the generation of $\mathrm{Ca}^{2+}$ transients in mouse embryos during the first mitotic division. Journal of Cell Biology 132 915-923.

Kouchi Z, Fukami K, Shikano T, Oda S, Nakamura Y, Takenawa T \& Miyazaki S 2004 Recombinant phospholipase Czeta has high $\mathrm{Ca}^{2+}$ sensitivity and induces $\mathrm{Ca}^{2+}$ oscillations in mouse eggs. Journal of Biological Chemistry 279 10408-10412.

Kuroda K, Ito M, Shikano T, Awaji T, Yoda A, Takeuchi H, Kinoshita K \& Miyazaki S 2006 The role of $X / Y$ linker region and N-terminal EF-hand domain in nuclear translocation and $\mathrm{Ca}^{2+}$ oscillation-inducing activities of phospholipase czeta, a mammalian egg-activating factor. Journal of Biological Chemistry 281 27794-27805.

Kurokawa M \& Fissore RA 2003 ICSI-generated mouse zygotes exhibit altered calcium oscillations, inositol 1,4,5-trisphosphate receptor-1 down-regulation, and embryo development. Molecular Human Reproduction 9 523-533.

Kurokawa M, Sato K, Smyth J, Wu H, Fukami K, Takenawa T \& Fissore RA 2004 Evidence that activation of Src family kinase is not required for fertilization-associated $\left[\mathrm{Ca}^{2+}\right]$ i oscillations in mouse eggs. Reproduction 127 441-454.

Kurokawa M, Sato K, Wu H, He C, Malcuit C, Black SJ, Fukami K \& Fissore RA 2005 Functional, biochemical, and chromatographic characterization of the complete $\left[\mathrm{Ca}^{2+}\right](\mathrm{i})$ oscillation-inducing activity of porcine sperm. Developmental Biology 285 376-392.

Kyozuka K, Deguchi R, Mohri T \& Miyazaki S 1998 Injection of sperm extract mimics spatiotemporal dynamics of $\mathrm{Ca}^{2+}$ responses and progression of meiosis at fertilization of ascidian oocytes. Development 125 4099-4105. 
Larman MG, Saunders CM, Carroll J, Lai FA \& Swann K 2004 Cell cycledependent $\mathrm{Ca}^{2+}$ oscillations in mouse embryos are regulated by nuclear targeting of PLCzeta. Journal of Cell Science 117 2513-2521.

Lawrence Y, Whitaker M \& Swann K 1997 Sperm-egg fusion is the prelude to the initial $\mathrm{Ca}^{2+}$ increase at fertilization in the mouse. Development 124 233-241.

Lee SJ \& Shen SS 1998 The calcium transient in sea urchin eggs during fertilization requires the production of inositol 1,4,5-trisphosphate. Developmental Biology 193 195-208.

Lee B, Yoon SY \& Fissore RA 2006 Regulation of fertilization-initiated $\left[\mathrm{Ca}^{2+}\right] \mathrm{i}$ oscillations in mammalian eggs: a multi-pronged approach. Seminars in Cell and Developmental Biology 17 274-284.

Malcuit C, Knott JG, He C, Wainwright T, Parys JB, Robl JM \& Fissore RA 2005 Fertilization and inositol 1,4,5-trisphosphate (IP3)-induced calcium release in type-1 inositol 1,4,5-trisphosphate receptor down-regulated bovine eggs. Biology of Reproduction 73 2-13.

Manandhar G \& Toshimori K 2003 Fate of postacrosomal perinuclear theca recognized by monoclonal antibody $\mathrm{MN} 13$ after sperm head microinjection and its role in oocyte activation in mice. Biology of Reproduction 68 655-663.

Marangos P, FitzHarris G \& Carroll J $2003 \mathrm{Ca}^{2+}$ oscillations at fertilization in mammals are regulated by the formation of pronuclei. Development 130 1461-1472.

Miyazaki S, Yuzaki M, Nakada K, Shirakawa H, Nakanishi S, Nakade S \& Mikoshiba K 1992 Block of $\mathrm{Ca}^{2+}$ wave and $\mathrm{Ca}^{2+}$ oscillation by antibody to the inositol 1,4,5-trisphosphate receptor in fertilized hamster eggs. Science 257 251-255.

Moses RM \& Kline D 1995 Calcium-independent, meiotic spindledependent metaphase-to-interphase transition in phorbol ester-treated mouse eggs. Developmental Biology 171 111-122.

Moos J, Visconti PE, Moore GD, Schultz RM \& Kopf GS 1995 Potential role of mitogen-activated protein kinase in pronuclear envelope assembly and disassembly following fertilization of mouse eggs. Biology of Reproduction 53 692-699.

Nakano Y, Shirakawa H, Mitsuhashi N, Kuwabara Y \& Miyazaki S 1997 Spatiotemporal dynamics of intracellular calcium in the mouse egg injected with a spermatozoon. Molecular Human Reproduction 3 1087-1093.

Ogonuki N, Sankai T, Yagami K, Shikano T, Oda S, Miyazaki S \& Ogura A 2001 Activity of a sperm-borne oocyte-activating factor in spermatozoa and spermatogenic cells from cynomolgus monkeys and its localization after oocyte activation. Biology of Reproduction 65 351-357.

Ozil JP, Markoulaki S, Toth S, Matson S, Banrezes B, Knott JG, Schultz RM, Huneau D \& Ducibella T 2005 Egg activation events are regulated by the duration of a sustained $\left[\mathrm{Ca}^{2+}\right]_{\text {cyt }}$ signal in the mouse. Developmental Biology 282 39-54.

Palermo G, Joris H, Devroey P \& Van Steirteghem AC 1992 Pregnancies after intracytoplasmic injection of single spermatozoon into an oocyte. Lancet 340 17-18.

Parrington J, Lai FA \& Swann K 2000 The soluble mammalian sperm factor protein that triggers $\mathrm{Ca}^{2+}$ oscillations in eggs: evidence for expression of mRNA(s) coding for sperm factor protein(s) in spermatogenic cells. Biology of the Cell 92 267-275.

Parrish JJ, Krogenaes A \& Susko-Parrish JL 1995 Effect of bovine sperm separation by either swim-up or Percoll method on success of in vitro fertilization and early embryonic development. Theriogenology 44 859-869.

Perry AC, Wakayama T \& Yanagimachi R 1999 A novel trans-complementation assay suggests full mammalian oocyte activation is coordinately initiated by multiple, submembrane sperm components. Biology of Reproduction 60 747-755.

Perry AC, Wakayama T, Cooke IM \& Yanagimachi R 2000 Mammalian oocyte activation by the synergistic action of discrete sperm head components: induction of calcium transients and involvement of proteolysis. Developmental Biology 217 386-393.

Rhee SG \& Bae YS 1997 Regulation of phosphoinositide-specific phospholipase C isozymes. Journal of Biological Chemistry 272 15045-15048.

Rogers NT, Hobson E, Pickering S, Lai FA, Braude P \& Swann K 2004 Phospholipase Czeta causes $\mathrm{Ca}^{2+}$ oscillations and parthenogenetic activation of human oocytes. Reproduction 128 697-702.
Sato MS, Yoshitomo M, Mohri T \& Miyazaki S 1999 Spatiotemporal analysis of $\left[\mathrm{Ca}^{2+}\right]_{i}$ rises in mouse eggs after intracytoplasmic sperm injection (ICSI). Cell Calcium 26 49-58.

Saunders CM, Larman MG, Parrington J, Cox LJ, Royse J, Blayney LM, Swann K \& Lai FA 2002 PLC zeta: a sperm-specific trigger of $\mathrm{Ca}^{2+}$ oscillations in eggs and embryo development. Development 129 3533-3544.

Schultz RM \& Kopf GS 1995 Molecular basis of mammalian egg activation. Current Topics in Developmental Biology 30 21-62.

Sone Y, Ito M, Shirakawa H, Shikano T, Takeuchi H, Kinoshita K \& Miyazaki S 2005 Nuclear translocation of phospholipase C-zeta, an eggactivating factor, during early embryonic development. Biochemical and Biophysical Research Communications 330 690-694.

Stricker SA 1997 Intracellular injections of a soluble sperm factor trigger calcium oscillations and meiotic maturation in unfertilized oocytes of a marine worm. Developmental Biology 186 185-201.

Stricker SA 1999 Comparative biology of calcium signaling during fertilization and egg activation in animals. Developmental Biology 211 157-176.

Sutovsky P, Manandhar G, Wu A \& Oko R 2003 Interactions of sperm perinuclear theca with the oocyte: implications for oocyte activation, anti-polyspermy defense, and assisted reproduction. Microscopic Research and Technique 61 362-378.

Swann K 1990 A cytosolic sperm factor stimulates repetitive calcium increases and mimics fertilization in hamster eggs. Development 110 1295-1302.

Swann K 1996 Soluble sperm factors and $\mathrm{Ca}^{2+}$ release in eggs at fertilization. Reviews of Reproduction 1 33-39.

Swann K, Larman MG, Saunders CM \& Lai FA 2004 The cytosolic sperm factor that triggers $\mathrm{Ca}^{2+}$ oscillations and egg activation in mammals is a novel phospholipase C: PLCzeta. Reproduction 127 431-439.

Swann K, Saunders CM, Rogers NT \& Lai FA 2006 PLCzeta (zeta): a sperm protein that triggers $\mathrm{Ca}^{2+}$ oscillations and egg activation in mammals. Seminars in Cell and Developmental Biology 17 264-273.

Tang TS, Dong JB, Huang XY \& Sun FZ $2000 \mathrm{Ca}^{2+}$ oscillations induced by a cytosolic sperm factor are mediated by a maternal machinery that functions only once in mammalian eggs. Development 127 1141-1150.

Wu H, He CL \& Fissore RA 1997 Injection of a porcine sperm factor triggers calcium oscillations in mouse oocytes and bovine eggs. Molecular Reproduction and Development 46 176-189.

Wu H, Smyth J, Luzzi V, Fukami K, Takenawa T, Black SL, Allbritton NL \& Fissore RA 2001 Sperm factor induces intracellular free calcium oscillations by stimulating the phosphoinositide pathway. Biology of Reproduction 64 1338-1349.

Xu Z, Kopf GS \& Schultz RM 1994 Involvement of inositol 1,4,5trisphosphate-mediated $\mathrm{Ca}^{2+}$ release in early and late events of mouse egg activation. Development 120 1851-1859.

Yamamoto S, Kubota HY, Yoshimoto Y \& Iwao Y 2001 Injection of a sperm extract triggers egg activation in the newt Cynops pyrrhogaster. Developmental Biology 230 89-99.

Yazawa H, Yanagida K, Katayose H, Hayashi S \& Sato A 2000 Comparison of oocyte activation and $\mathrm{Ca}^{2+}$ oscillation-inducing abilities of round/elongated spermatids of mouse, hamster, rat, rabbit and human assessed by mouse oocyte activation assay. Human Reproduction 15 2582-2590.

Yoda A, Oda S, Shikano T, Kouchi Z, Awaji T, Shirakawa H, Kinoshita K \& Miyazaki S $2004 \mathrm{Ca}^{2+}$ oscillation-inducing phospholipase C zeta expressed in mouse eggs is accumulated to the pronucleus during egg activation. Developmental Biology 268 245-257.

Yoneda A, Kashima M, Yoshida S, Terada K, Nakagawa S, Sakamoto A, Hayakawa K, Suzuki K, Ueda J \& Watanabe T 2006 Molecular cloning, testicular postnatal expression, and oocyte-activating potential of porcine phospholipase Czeta. Reproduction 132 393-401.

Received 5 June 2007

First decision 25 June 2007

Accepted 8 August 2007 